\title{
Diabetic Peripheral Neuropathy in Ethiopia: A Systematic Review and Meta-Analysis
}

\author{
Degena Bahrey Tadesse $\mathbb{D}^{1},{ }^{1}$ Gebremeskel Tukue Gebrewahd $\left(\mathbb{D},{ }^{2}\right.$ Abrha Hailay $\mathbb{D}^{1}$ \\ Woldu Aberhe, ${ }^{1}$ Guesh Mebrahtom ${ }^{\mathbb{D}},{ }^{1}$ Kidane Zereabruk, ${ }^{1}$ Guesh Gebreayezgi $\mathbb{D}^{3}{ }^{3}$ \\ Teklewoini Mariye $\mathbb{D}^{1}{ }^{1}$ Teklehaimanot Gereziher Haile, ${ }^{4}$ \\ Gebreamlak Gebremedhn Gebremeskel, ${ }^{1}$ and Gebre Teklemariam Demoz ${ }^{5}{ }^{5}$
}

\author{
${ }^{1}$ Department of Adult Health Nursing, School of Nursing, Aksum University, Aksum, Ethiopia \\ ${ }^{2}$ Department of Emergency Nursing, School of Nursing, Aksum University, Aksum, Ethiopia \\ ${ }^{3}$ Department of Epidemiology, School of Public Health, Aksum University, Aksum, Ethiopia \\ ${ }^{4}$ Department of Maternal and Neonatal Nursing, School of Public Health, Aksum University, Aksum, Ethiopia \\ ${ }^{5}$ Department of Clinical Pharmacy, School of Pharmacy, Aksum University, Aksum, Ethiopia
}

Correspondence should be addressed to Degena Bahrey Tadesse; shewitdege@gmail.com

Received 31 March 2020; Revised 31 October 2020; Accepted 2 November 2020; Published 5 February 2021

Academic Editor: Claudia Cardoso

Copyright (C) 2021 Degena Bahrey Tadesse et al. This is an open access article distributed under the Creative Commons Attribution License, which permits unrestricted use, distribution, and reproduction in any medium, provided the original work is properly cited.

Background. Currently, diabetic peripheral neuropathy (DPN) is one of the most severe complications of diabetes mellitus (DM). Despite the seriousness of this problem, limited evidence is available on the prevalence of diabetic peripheral neuropathy among patients with diabetes mellitus in Ethiopia. In Ethiopia, there were no updated studies that estimate the national prevalence of DPN. Hence, this systematic review and meta-analysis provided a national prevalence of diabetic peripheral neuropathy among patients with diabetes mellitus in Ethiopia. Methods. This study was submitted for registration with the International Prospective Register of Systematic Reviews (PROSPERO) in March 2020 and accepted with the registration number CRD42020173831. Different database searching engines were searched online to retrieve related articles, including PubMed, Scopus, Google Scholar, African Journals Online, World Health Organization (WHO) Afro Library, and Cochrane Review. The reviewers used the Preferred Reporting Items for Systematic Review and Meta-Analysis (PRISMA) guideline in the reviewing process. In this systematic review and meta-analysis, all published and unpublished articles were analyzed. The reviewers used the random effects model to estimate the pooled prevalence of diabetic peripheral neuropathy among diabetes mellitus patients. The reviewers conducted the statistical analysis using the $\mathrm{R}$ version 3.5.3 and RStudio version 1.2.5033 software for Windows. The reviewers evaluated the heterogeneity across the included studies by the inconsistency index $\left(I^{2}\right)$. The reviewers examined the publication bias by the funnel plot. Results. The search of the databases produced 245 papers. After checking the inclusion and exclusion criteria, 38 articles with 14029 total patients with diabetes mellitus were found suitable for the review. Except for three (retrospective cohort study), all studies were cross-sectional. The overall pooled prevalence of diabetic peripheral neuropathy was $22 \%$ (95\% CI $18 \%$ to $26 \%$ ). The subgroup analysis of diabetic peripheral neuropathy among patients with diabetes in the different regions was $23 \%$ (95\% CI $17 \%$ to $29 \%$ ) in Addis Ababa, $27 \%$ (95\% CI $16 \%$ to $38 \%$ ) in Oromia, $16 \%$ (95\% CI 14\% to 18\%) in South nation and nationalities, and 15\% (95\% CI 6\% to 24\%) in Amhara. Conclusions. More than onefifth of patients with diabetes have diabetic peripheral neuropathy. According to this study, the prevalence of diabetic peripheral neuropathy in Ethiopia is considerably high. This evidence suggests that attention should be given to patients with diabetes in monitoring patients' blood glucose. 


\section{Introduction}

Currently, half a billion people are living with diabetes worldwide and will increase by 578 million (25\%) in 2030 and 700 million (51\%) in 2045 [1].

Diabetes mellitus (DM) is a metabolic disorder that can predispose to diseases of the heart, blood vessels, lungs, kidneys, and nerves. Such damage may result in a decreased blood flow to the feet, which is coupled with neuropathy to the nerves-raising the risk of foot ulcers, inflammation, and subsequent limb amputation [2].

One of the most severe complications of DM is diabetic polyneuropathy (DPN). The lifetime prevalence of DPN was $50 \%$ [3-5]. DPN is associated with a significant reduction in quality of life and poses treatment challenges to the practicing physician [4]. It is also the cause of impairment due to foot ulceration and amputation, disruption of the gait, and damage involved with dropping. About 20 to 30 percent of DPN patients suffer from neuropathic pain [4-6].

The average estimated expense for diabetes medication, health insurance of DPN, and debilitating DPN is \$6632, \$12492, and \$30755, respectively [7].

In Ethiopia, the prevalence of DPN is estimated to be in the range of $1.9-53.6 \%$ that shows a difference across different geographical settings and time $[8,9]$. Many factors contribute to this huge difference, including study quality, study design, ethnic differences, and different diagnostic methods. Reliable estimates of DPN prevalence are required to enhance awareness of the prevention and management of DPN in patients with diabetes. Therefore, the primary purpose of this systematic review and meta-analysis is to determine the prevalence of DPN among patients with diabetes mellitus in Ethiopia.

\section{Materials and Methods}

2.1. Setting. Ethiopia is an East African country containing ten regions, namely, Tigray; Afar; Amhara; Oromia; Somali; Benishangul-Gumuz; Southern Nations, Nationalities, and People's Region (SNNPR), Gambella; Sidama Harari; and two urban administrative states (Addis Ababa City administration and Dire Dawa City administration).

2.2. Search Strategy and Information Sources. A search strategy was implemented using electronic databases (PubMed/MEDLINE, Embase, Google Scholar, Web of Science, Cochran Library, Africa-Wide Information, World Health Organization (WHO) Afro Library, and African Index Medicus) from inception to October 2020.

The reviewers have checked the presence of a precursor systematic review and protocol on the topic of interest via searching for different databases. The included databases were the Cochrane Database of Systematic Reviews, Joanna Briggs Institute Database of Systematic Review and Implementation Reports (JBI-DSRIR), the national health center review and dissemination database, health technology assessment (HTA), the Campbell Collaboration Library, and Evidence for Policy and Practice Information (EPPI-Centre).
The reviewers have developed a literature search technique using the headings of the medical subject headings (Met) and Boolean (AND/OR) operator. The reviewers have used the combination of key terms "DPN", "Diabetic peripheral neuropathy", "Diabetes mellitus", "Diabetic complication", "Macro and micro-vascular diabetic complication", "diabetic polyneuropathy", "Ethiopia", "systematic review", and protocols. The search from the above databases confirmed that there was no systematic review and/or protocol on the topic of interest.

2.3. Data Extraction, Selection, and Process. Based on the inclusion and exclusion criteria, the reviewers have developed a tool to guide the screening and selection process. This tool was piloted and revised before data extraction begins. The search results were first uploaded to the EndNote software to remove duplicates.

Two blind and independent reviewers extracted the data using a preconceived and standardized data collection format. The two reviewers (DBT and GGG) screened the titles, abstracts, and full-text search results to identify potentially eligible studies. Where necessary, the authors were contacted for additional information to confirm the eligibility of the studies. Disagreements between the reviewers were resolved by discussion with the help of a third independent reviewer. Where there is missing information, the reviewers contacted the corresponding author of the study to request the missing data. A maximum of three emails was sent to the corresponding author of the retrieved studies to request additional information before excluding the article. For studies appearing in more than one publication, we considered the most recent and comprehensive studies and those with the largest sample size. For surveys appearing in one article with multiple surveys conducted at different time points, we treated each as a separate study.

The components of the data extraction format included the information on the year of publication, region, authors, and country; setting, objective, and study design; diagnostic criteria of DPN; prevalence; or incidence of DPN.

\subsection{Criteria for Considering Studies for the Review}

2.4.1. Inclusion Criteria. The inclusion criteria are as follows: Design: all observational studies.

Population: study participants of at least 18 years of age with type 1 or $2 \mathrm{DM}$.

Publication status: published and unpublished studies.

Settings: all health institution-based studies.

Language: articles in the English language.

Publication or report year: studies conducted from 1976 to 2020.

Method of diagnosis: all studies, regardless of the method used for diagnosing DPN.

Intervention(s)/exposure(s): patients taking any form of antidiabetic medication.

Outcome: the primary outcome is the prevalence of DPN among diabetes mellitus patients in Ethiopia. It is defined by international consensus guidelines as "the presence of 
symptoms and/or signs of peripheral nerve dysfunction in people with diabetes after exclusion of other causes" $[10,11]$.

2.4.2. Exclusion Criteria. The exclusion criteria are as follows: case report and case series studies, studies that lack relevant data needed to compute the prevalence of DPN, studies on pregnancy-related diabetes mellitus, and studies on children and adolescents $<18$ years.

2.5. Risk of Bias and Quality Assessment of the Included Studies. Methodological quality and risk of bias assessments were performed by two reviewers (DBT and GGG), blindly and independently. The reviewers maintained the blinding reviewing method using the Covidence software that allows/obligates each reviewer to work without knowing the other reviewer's choice. This helps to diminish errors and the risk of bias in the selection of the studies. Disagreements between the reviewers were resolved by discussion and where necessary involving a third author.

For each included study, we estimated the precision $(C)$ or margin of error, considering the sample size (SS) and the observed prevalence $(p)$ of DPN using the formula:

$$
\mathrm{SS}=\frac{z^{2} * p *(1-p)}{d^{2}}
$$

where $z$ was the $z$ value fixed at 1.96 across studies (corresponding to $95 \%$ confidence interval). The desirable margin of error is $5 \%(0.05)$ or lower.

The methodological quality was evaluated using the Newcastle-Ottawa Scale. The scale is primarily formulated by a star allocation system, assigning a maximum of 10 stars for the risk of bias in three areas: a selection of study groups (4 or 5 stars), comparability of groups (2 stars), and ascertainment of the outcome of interest or the exposure (3 stars). No validation study provides a cutoff score for rating lowquality studies. We arbitrarily established 0-3, 4-6, and 710 stars considered at high, moderate, and low risk of bias, respectively [12].

2.6. Data Analysis and Presentation of Results. The reviewers have registered this review in PROSPERO with the registration number (CRD42020173831), and the Preferred Reporting Items for Systematic Reviews and Meta-Analyses (PRISMA) guidelines have been used to report the results [13]. The data were analyzed using the $\mathrm{R}$ version 3.5.3 and RStudio version 1.2.5003 software. The pooled prevalence of diabetic peripheral neuropathy was estimated using forest plots and the extent of statistical heterogeneity between studies. Statistical heterogeneity was assessed using the standard chi-squared test (Cochran $Q$ test) and quantified by calculating the $I^{2}$ statistics (with values of $25 \%, 50 \%$, and $75 \%$ assumed to be representative of low, medium, and high heterogeneity, respectively) [14]. There was clinical heterogeneity between the included studies. Consequently, we used a random effects meta-analysis to estimate the overall pooled prevalence of DPN in Ethiopia. The reviewers used a funnel plot to evaluate publication bias and the $95 \%$ confidence interval $(\mathrm{CI})$ to describe the results.

\section{Results}

3.1. Screening Flow. Figure 1 is a flow diagram outlining the process of identification and selection of the included studies. The included databases and number of included studies thereof were PubMed (63), Scopus (46), Google Scholar (107), and the World Health Organization (WHO) Afro Library (29). Of these studies, 101 are duplicates and removed. Subsequently, we screened 144 titles and abstracts and excluded 66 irrelevant papers. Then, based on the predefined criteria and quality assessment, 38 full-text articles with 14029 total patients with diabetes were included in this systematic review and meta-analysis. The detailed steps of the screening process are shown in a PRISMA flow chart (Figure 1).

3.2. Study Characteristics. More than one-third (16, 42.1\%) of the studies were from studies conducted in the capital city of Ethiopia (Addis Ababa), 9 (28.9\%) in Oromia, 7 (18.2\%) in Amhara, 2 (5.26\%) in South Nation and Nationalities, and 1 (2.6\%) in Tigray. Except for three studies (cohort), all the rest were cross-sectional. Of these studies, six studies were among patients with type 2 diabetes mellitus, and the rest were conducted on type 1 and type 2 diabetes mellitus. The quality of each primary study assessed using the Newcastle-Ottawa Scale shows no considerable risk. Therefore, all the included studies considered in this systematic review and meta-analysis were with a low risk of bias (Table 1).

3.3. The Pooled Prevalence of DPN. The pooled prevalence of DPN among patients with diabetes in Ethiopia is 22\% (95\% CI $18-26 \%)$. The heterogeneity test shown in $I^{2}$ with $98 \%$ indicates high heterogeneity. The prevalence of diabetic peripheral neuropathy is varying widely in the studies. This heterogeneity might be due to differences in the diagnostic criteria employed and the different defining criteria of DPN used (Figure 2).

A funnel test was used to test for publication bias. And it showed the presence of publication bias. Therefore, there are unpublished data that can modify the prevalence of DPN (Figure 3).

3.4. Subgroup Analysis of DPN by Region of the Country. Based on the subgroup analysis of DPN among patients with diabetes attending hospitals in Addis Ababa, the pooled point estimate was 23\% (with 95\% CI; 17 to 29) (Figure 4).

Based on a subgroup analysis of DPN among patients with diabetes attending Oromia's hospitals, the pooled point estimate was 27\% (with 95\% CI; 16 to 38) (Figure 5).

Based on the subgroup analysis of DPN among patients with diabetes attending South Nation and Nationalities' hospitals, the pooled point estimate was $16 \%$ (with $95 \% \mathrm{CI} ; 14$ to 18) (Figure 6).

Based on the subgroup analysis of DPN among patients with diabetes attending Amhara's hospitals, the pooled point estimate was $15 \%$ (with 95\% CI; 6 to 24) (Figure 7). 


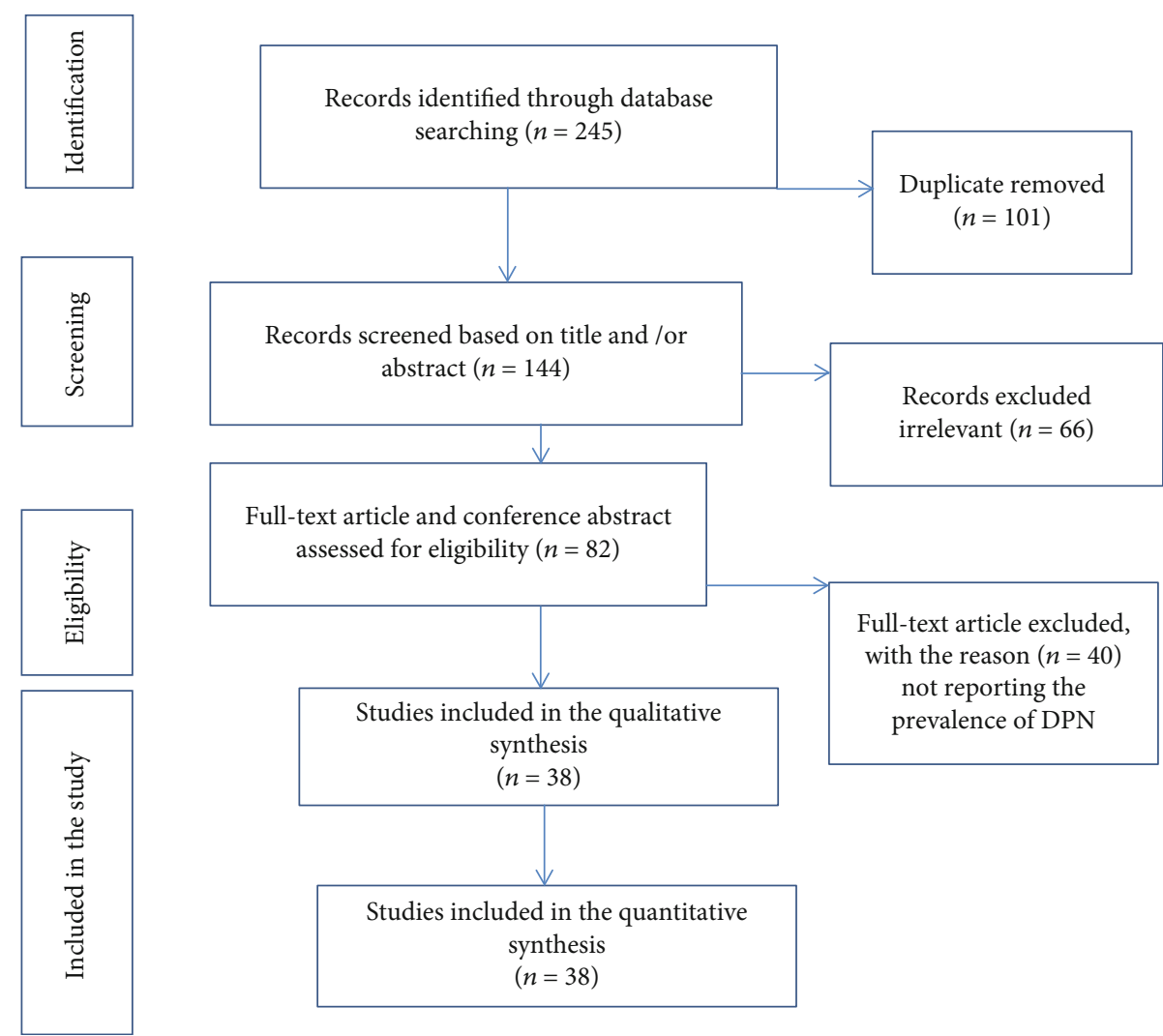

FIGURE 1: Flow chart diagram showing the selection of articles for systemic review and meta-analysis of DPN among patients with diabetes in Ethiopia, 2020.

\section{Discussion}

The reviewers have performed the present systematic review and meta-analysis to produce pooled estimates of nationwide results of peripheral neuropathy in Ethiopia among patients with diabetes. This review emphasized the burden of peripheral neuropathy among patients with diabetes in Ethiopia for a better understanding of the medical condition that can help in mitigating the problem of peripheral neuropathy in the country.

This meta-analysis showed the pooled estimate of diabetic peripheral neuropathy in Ethiopia to be standing at 22\% (with 95\% CI: 18-26). Individually, there was a variety in the prevalence of peripheral neuropathy in the studies included in our work which ranged from $1.9 \%$ as reported by Jember et al. [9] to $53.6 \%$ by Abdissa [47]. The reason for these variations could be due to the types of diabetes and assessment methods. The estimates of DPN are more common in patients with type 2 diabetes compared to those with type 1 diabetes (53\% in type 2 diabetes) in the metaanalysis reported from Iran [50] versus type 1 (28.2\% in type 1) [51]. As we estimated both in one, this truth is supported by one systematic review, reported in its subgroup analysis; the higher prevalence of peripheral neuropathy was lesser in patients with type 2 diabetes $(38.8 \%)$ than in patients with type 1 diabetes (17.5\%) [52]. The overall pooled prevalence of peripheral neuropathy in this study $(22 \%)$ was consistent with a study reported from Arab countries that had a pooled prevalence of diabetic peripheral neuropathy of $18 \%$ (with a 95\% CI: 0.09-0.34) [52]. Similarly, our finding was also comparable with a systematic review and meta-analysis reported from Oceania (23.2\%, 95\% CI: 2.68-76) [53]. This similarity might be due to the type of diabetes in our work conducted on both type 1 and type 2 diabetes, and the meta-analysis reported from Iran [52] focused on studies which included only patients with type 1 diabetes.

On the other hand, the result found in this study is lower as compared to other previous systematic review and metaanalysis studies that estimated the prevalence of peripheral neuropathy in patients with diabetes: $46 \%$ from the African region [54], 53\% from Iran [50], and 31.6\%, 32.2\%, and $48.1 \%$ from America, Asia, and Europe, respectively, with an overall prevalence of $35.7 \%$ [53]. Similarly, the result was smaller than the results found in other meta-analyses conducted among patients with type $1 \mathrm{DM}$ which showed a prevalence of $28.2 \%$ in Iran [51] and $30 \%$ in studies conducted in multination [55]. Our findings were higher compared with a worldwide estimate of DPN prevalence among patients with diabetes $(8.1 \%-12.2 \%)$ [56].

To look at the heterogeneity of the studies included in this review, we conducted subgroup analysis by regions, and we found that a study done in Addis Ababa had the highest pooled point estimate of peripheral neuropathy in patients with diabetes, accounting for $23 \%$ (95\% CI: 16, 30), and the lowest prevalence was reported from the Amhara region (15\%, 95\% CI: 6,24$)$. This discrepancy could be due 


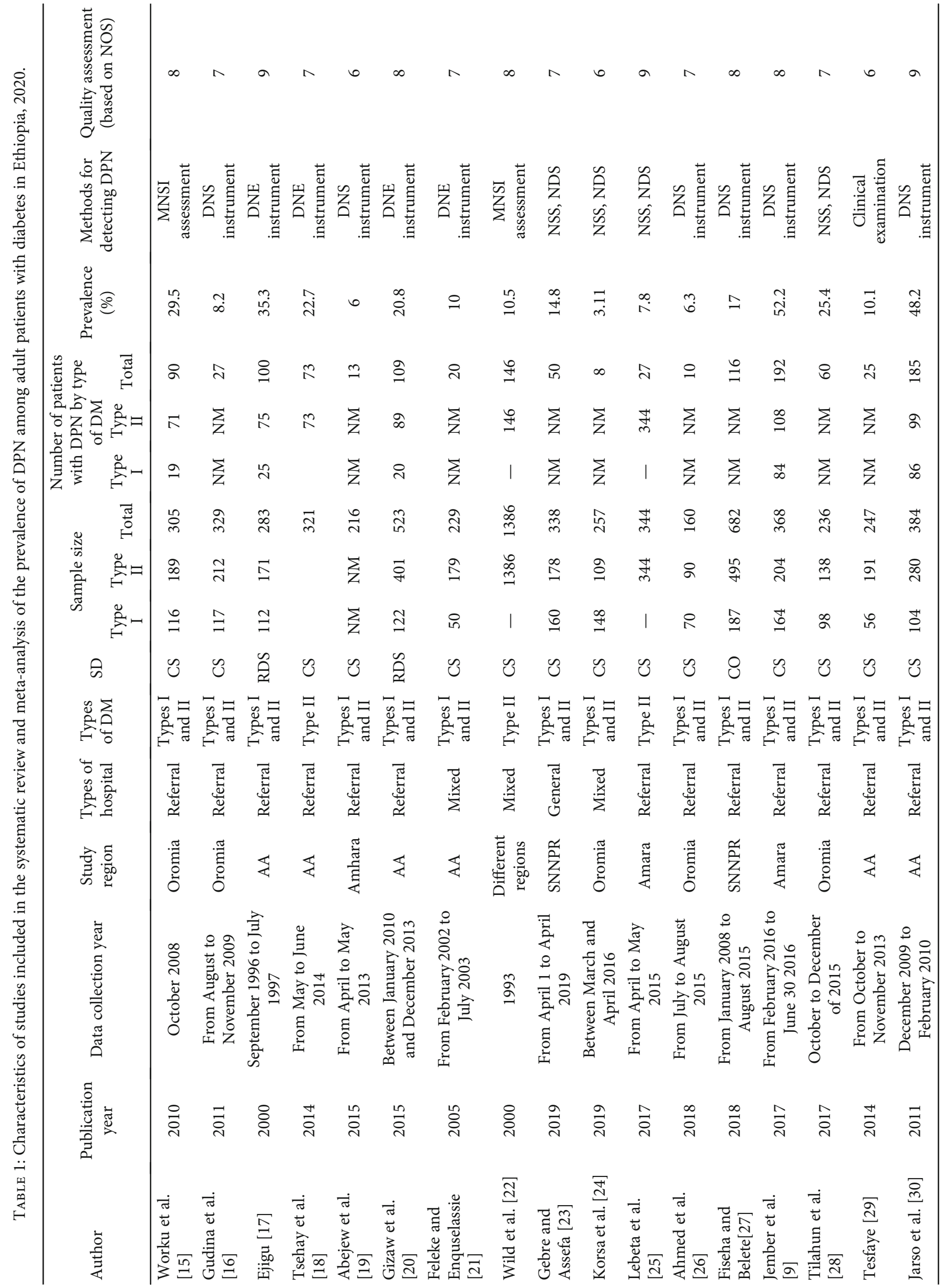




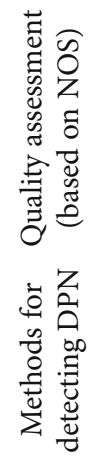

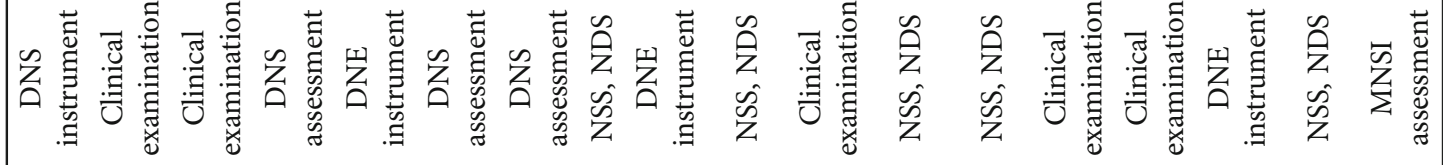

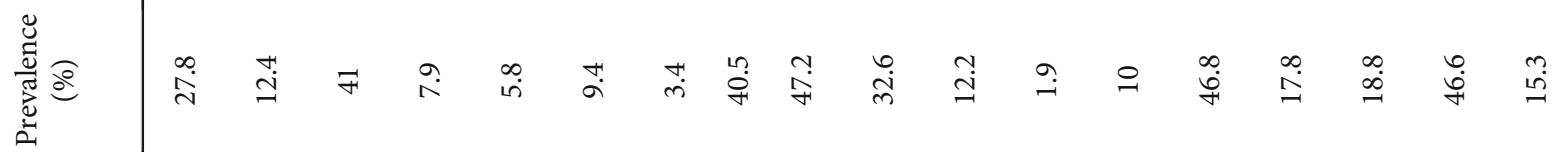
劳

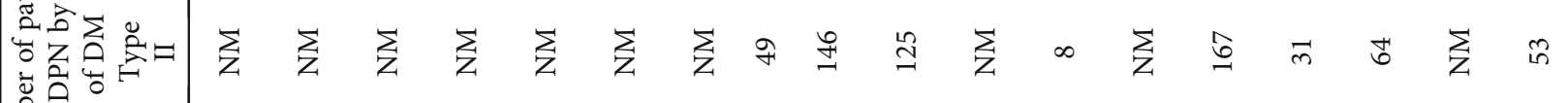

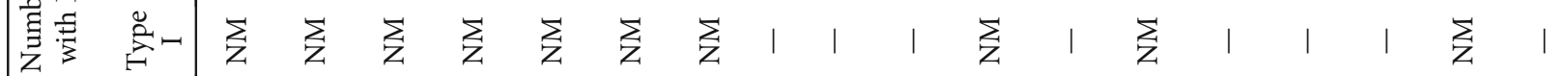
亚 के

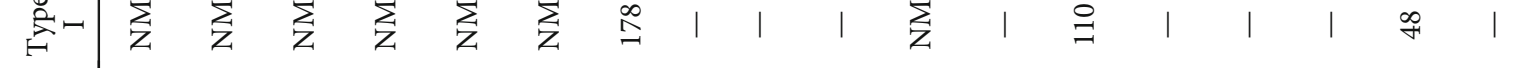

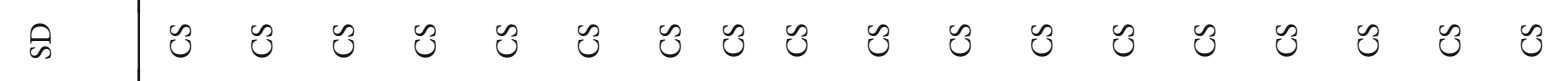

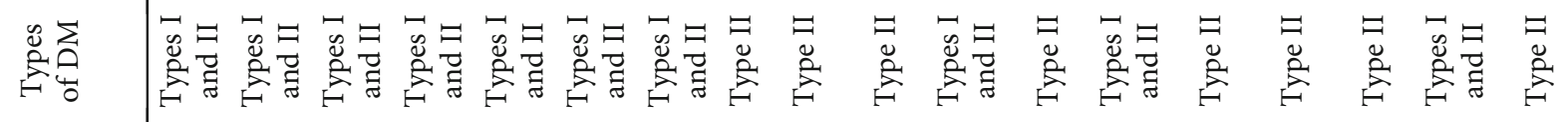

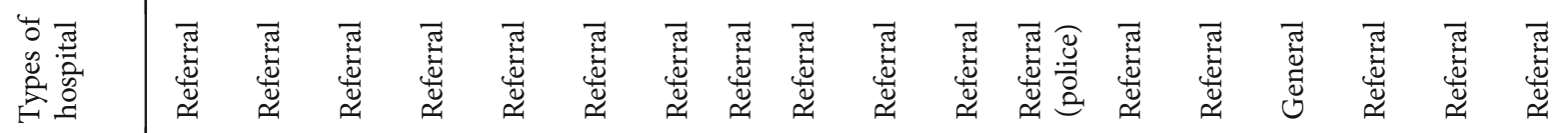

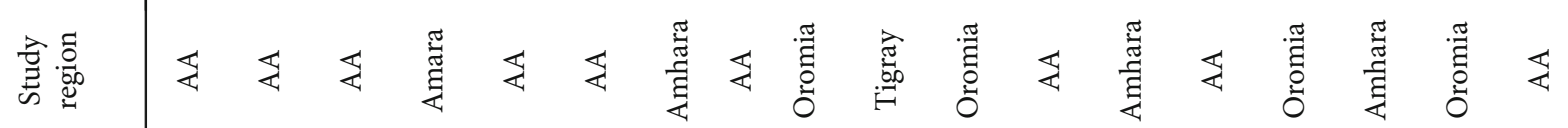

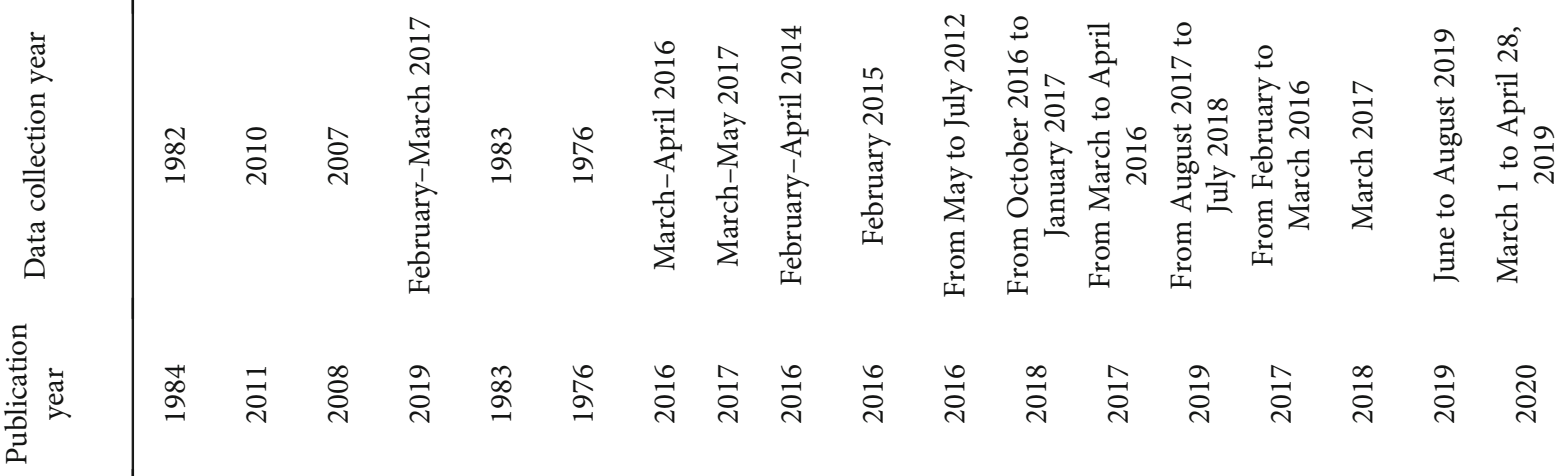

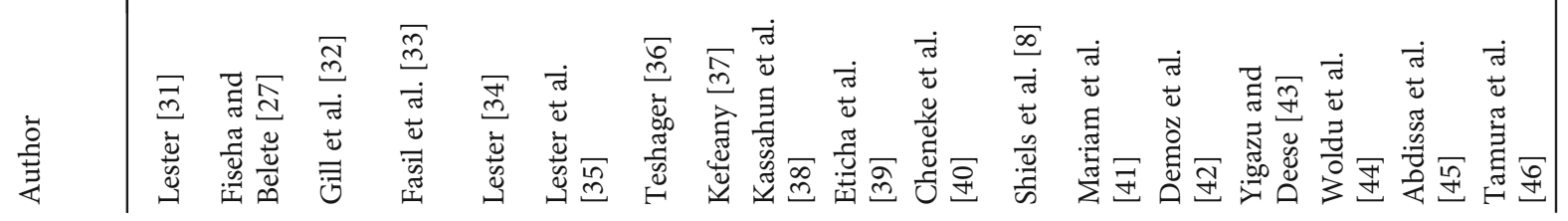




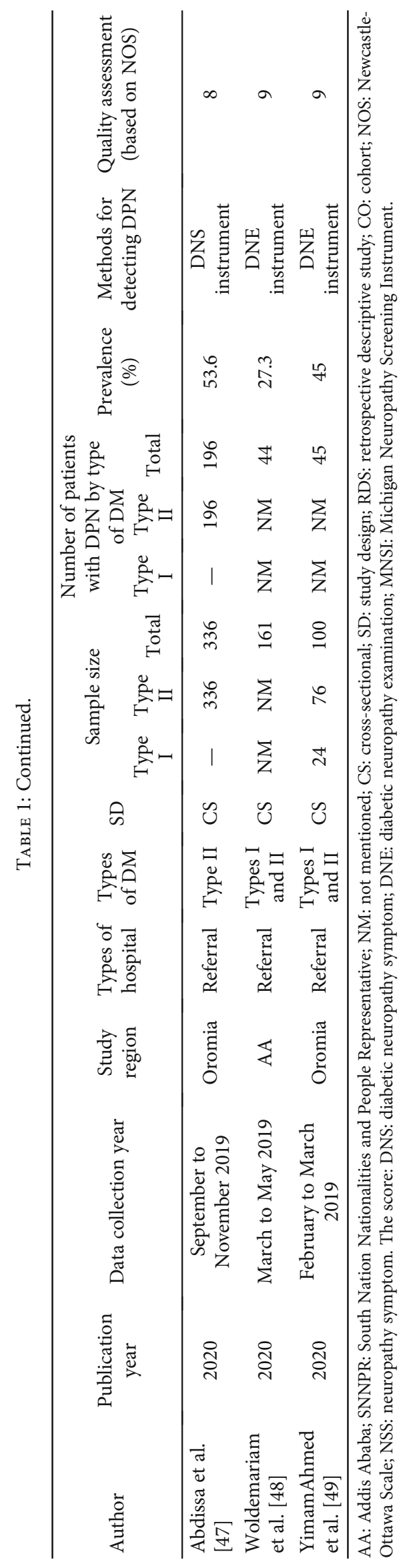




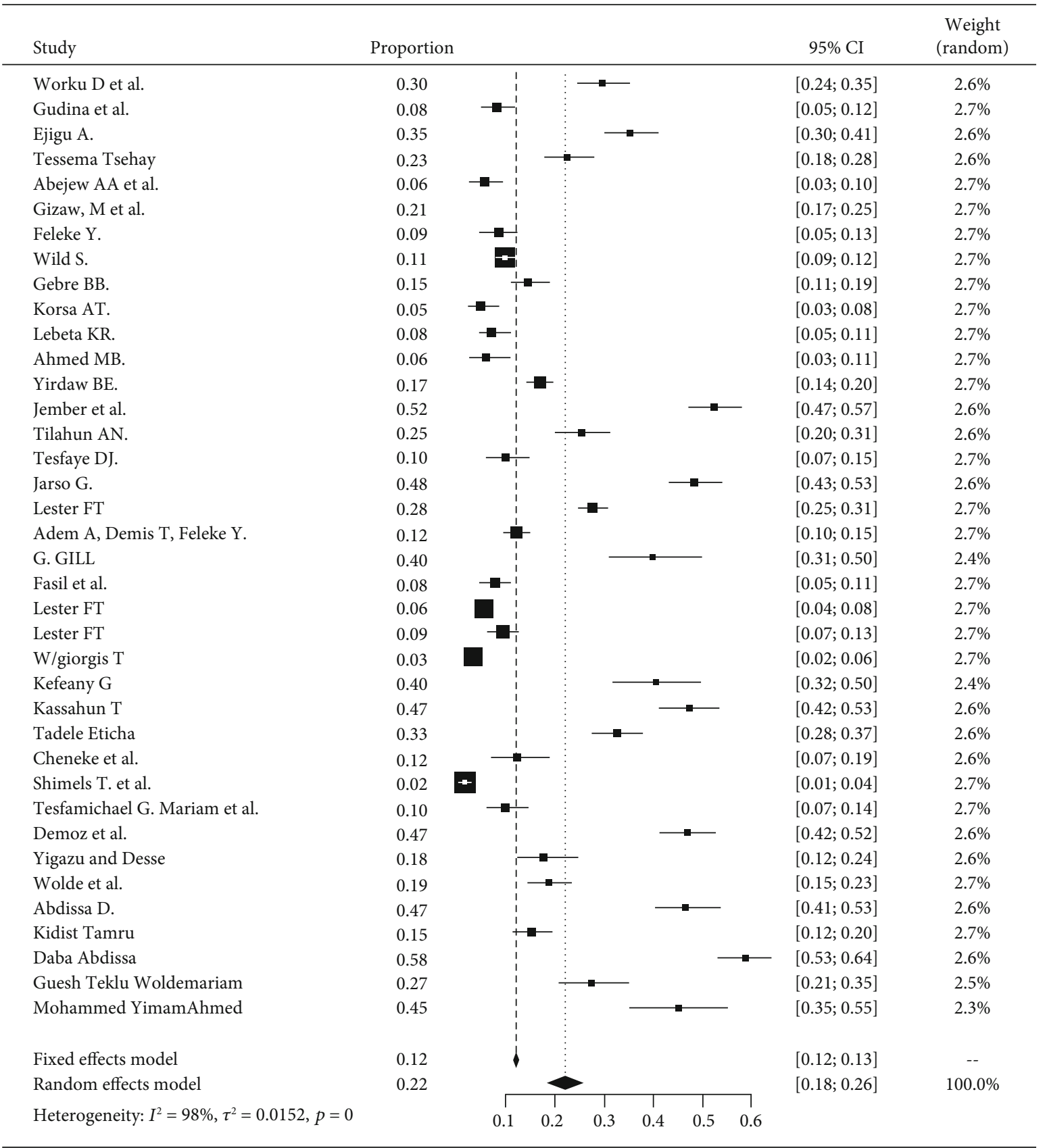

FIGURE 2: Forest plot for the pooled prevalence of DPN from 38 observational studies.

to the difference in health care settings such as tertiary and primary health care that may cause a difference in screening and assessment practice and quality of service for diabetes patients with peripheral neuropathy. Some health care facilities may be enriched with the specialty that works more on better assessment or good practice on prevention strategies or well found with diagnostic instruments and severity of diabetes. Moreover, it may also be because there are no uniform assessment criteria or diagnostic method in clinical practice in all health care settings or due to the use by different clinicians of their unique clinical expertise in examining and individualizing their patients.
Our findings from the meta-analysis have implications in clinical practice as it can contribute to giving attention to the prevention and care of patients with diabetes. This pooled point of estimates for peripheral neuropathy in patients with diabetes provides updated evidence to advance prevention strategy, serves as key indicators of patient safety, and reflects the quality of the health care service and appropriate treatment strategy for peripheral neuropathy in patients with diabetes. The finding of the prevalence of diabetic peripheral neuropathy improves with good glucose control; use of adherence interventions, such as patient education and counseling on how to selfmonitor blood glucose; and lifestyle modification 


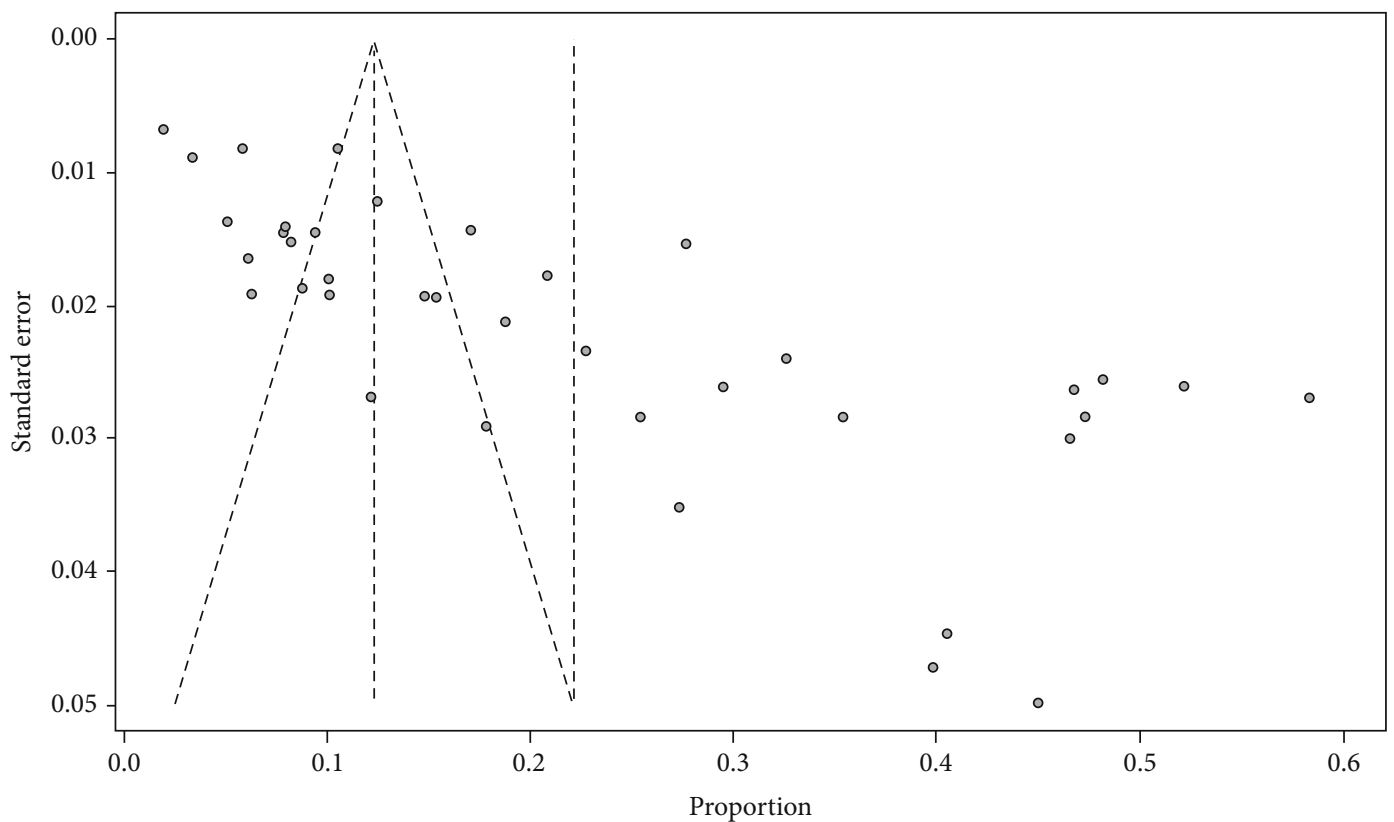

Figure 3: Funnel plot showing evidence of publication bias across studies.

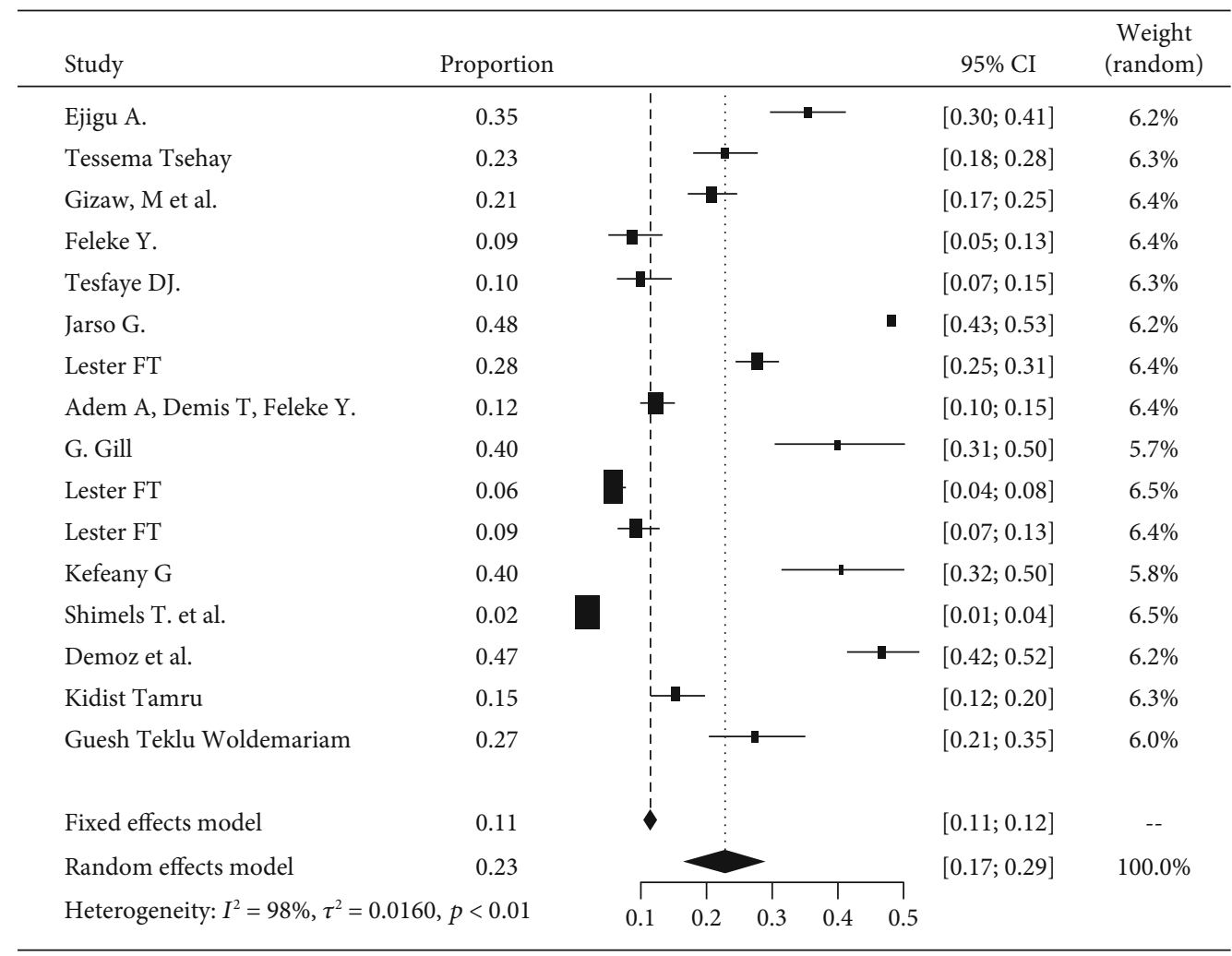

Figure 4: Forest plot of DPN in Addis Ababa.

interventions, such as exercise, weight reduction, and healthy diet. Therefore, the glycemic control strategy should be kept in mind that in addition to prescribing appropriate antidiabetic medicines and diabetes care practice, they need to include resources that help patients overcome individual challenges to reduce the development of such chronic diabetic complications using self-care practice. The implication of this study, particularly the pronounced variation between studies (1.9\% to 53.6\%), reflects patients with diabetes which require developing standards for management and implementing endorsed guidelines for clinical practice. 


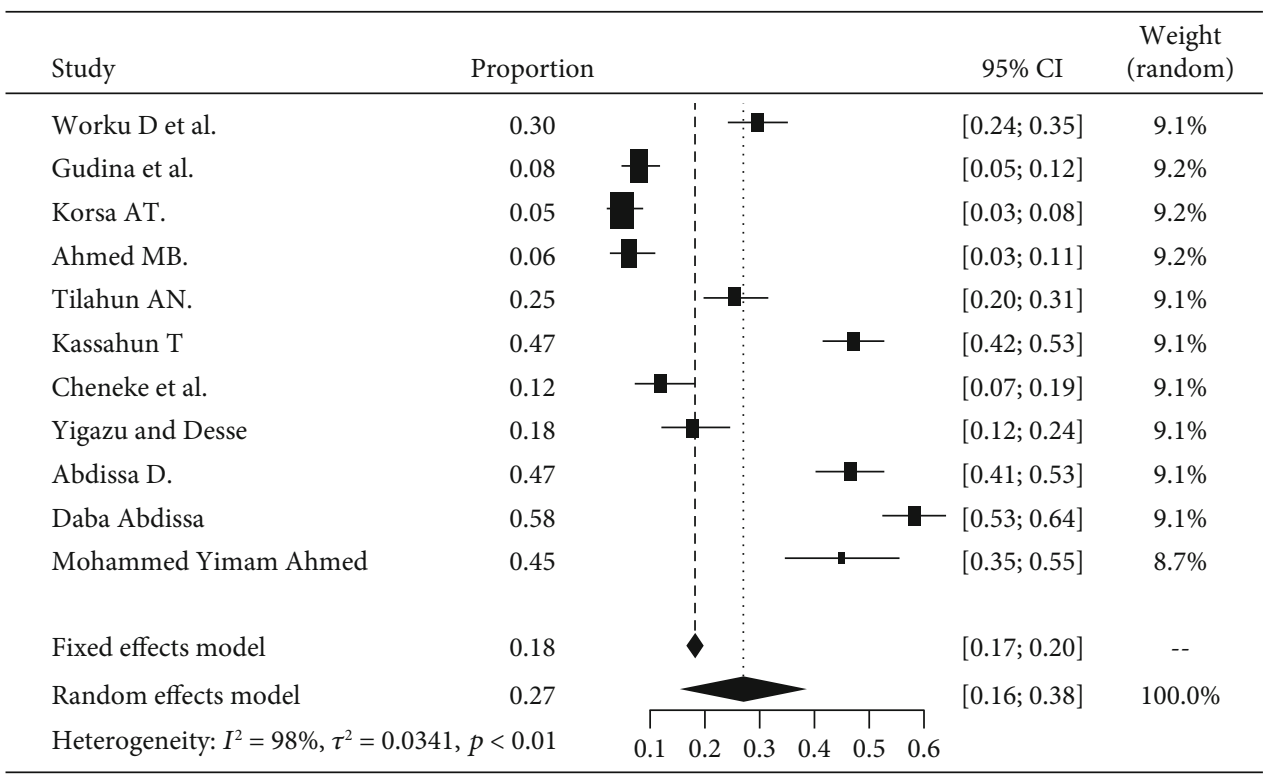

FIGURE 5: Forest plot of DPN in the Oromia region.

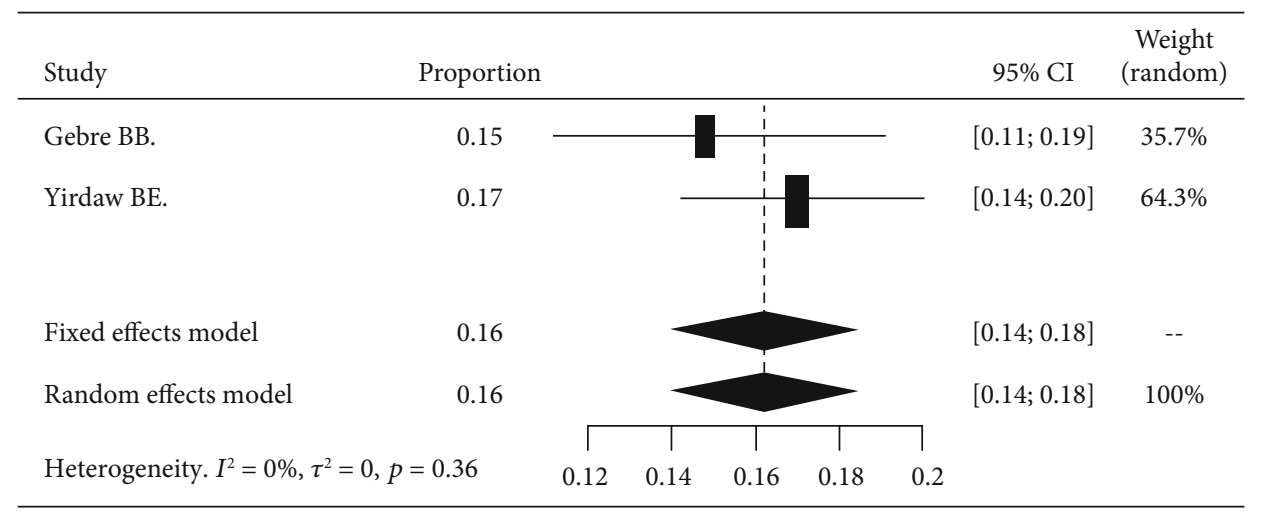

FIgURE 6: Forest plot of DPN in the south region.

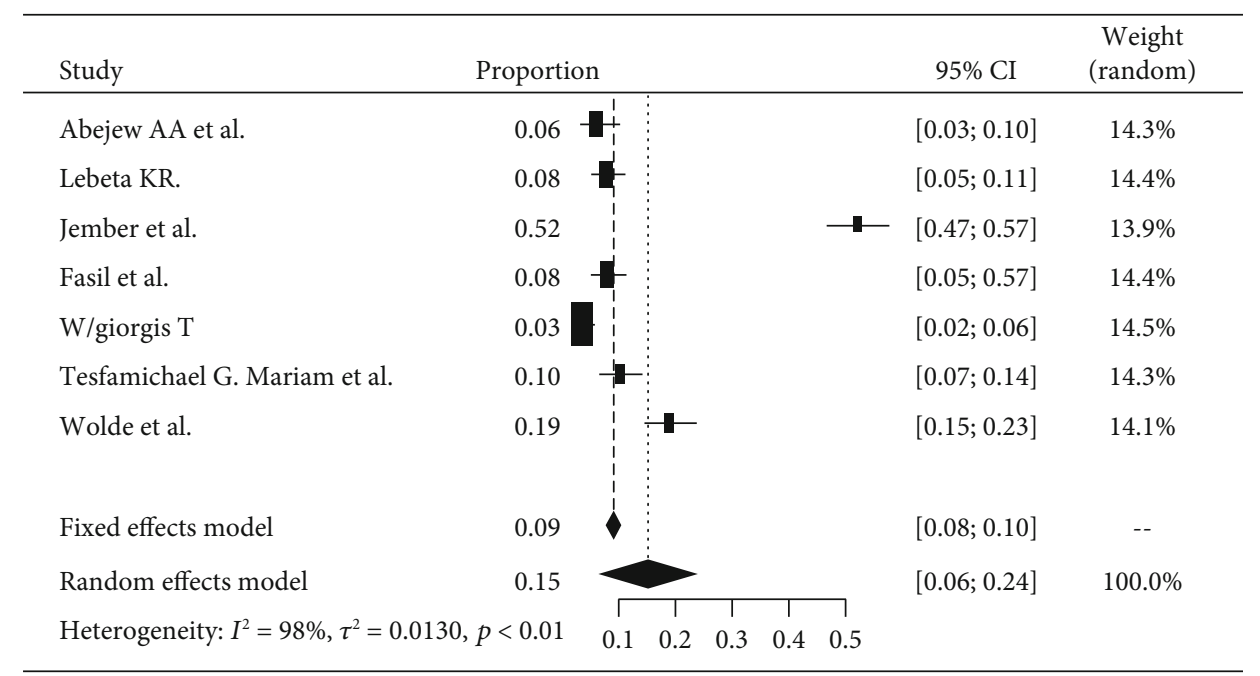

FIgURE 7: Forest plot of DPN in the Amhara region. 


\section{Limitations of This Study}

We conducted this review with rigor and standards of the art. So far, this is the first systematic review and meta-analysis that draws a clear picture of the pooled point of prevalence for peripheral neuropathy in patients with diabetes in Ethiopia. However, our work has certain limitations. Among these limitations are studies that did not describe the type of diabetes, and this could induce errors by finding untrue substantial associations when combining such studies affected by confounding. It was challenging to do a subgroup analysis for studies conducted among patients with type 1 and type 2 diabetes and those subjected to a high degree of heterogeneity among studies as we could not report by type of diabetes. But the random effects model was used to obtain the pooled results that can minimize such heterogeneity among studies. Additionally, the defining criteria for DPN differed from one to another study. This could also affect the results of the meta-analysis with far-reaching clinical heterogeneity across studies. The presence of publication bias was an additional limitation.

\section{Conclusion}

Our work systematically summarises the prevalence of peripheral neuropathy among patients with diabetes in Ethiopia that revealed that more than one-fifth of the patients with diabetes had developed peripheral neuropathy. Thus, our finding suggests that the prevalence of peripheral neuropathy is high at the national level but still lower than other previous studies reported from different countries. The Ministry of Health, health policymakers, clinicians, and other health care providers should strengthen the quality of health care service for patients with diabetes to reduce the development of peripheral neuropathy. The Ministry of Health should develop a context-based intervention and preventive strategies to reduce the burden of diabetes-related complications, particularly DPN.

\section{Abbreviations}

DM: $\quad$ Diabetes mellitus

DPN: $\quad$ Diabetic peripheral neuropathy

PRISMA-P: Preferred Reporting Items for Systematic reviews and Meta-Analysis protocol.

\section{Conflicts of Interest}

The authors declare that they have no conflicts of interest.

\section{Authors' Contributions}

DBT, GTG, AH, WA, TGH, and GTD developed the protocol and are involved in the design, selection of study, data extraction, statistical analysis, and development of the initial drafts of the manuscript. GG, GM, and KZ are involved in data extraction, quality assessment, statistical analysis, and revision. TM, DBT, GTD, and GGG prepared the final draft of the manuscript. All authors read and approved the final manuscript.

\section{Supplementary Materials}

The supplementary material includes the appendix about the search strategy and information sources. (Supplementary Materials)

\section{References}

[1] P. Saeedi, I. Petersohn, P. Salpea et al., "Global and regional diabetes prevalence estimates for 2019 and projections for 2030 and 2045: results from the International Diabetes Federation Diabetes Atlas, 9th edition," Diabetes Research and Clinical Practice, vol. 157, article 107843, 2019.

[2] G. Roglic, "WHO global report on diabetes: a summary," International Journal of Noncommunicable Diseases, vol. 1, no. 1, p. 3, 2016.

[3] R. Singh, L. Kishore, and N. Kaur, "Diabetic peripheral neuropathy: current perspective and future directions," Pharmacological Research, vol. 80, pp. 21-35, 2014.

[4] S. Tesfaye, L. Vileikyte, G. Rayman et al., "Painful diabetic peripheral neuropathy: consensus recommendations on diagnosis, assessment and management," Diabetes/Metabolism Research and Reviews, vol. 27, no. 7, pp. 629-638, 2011.

[5] S. Tesfaye, A. J. Boulton, P. J. Dyck et al., "Diabetic neuropathies: update on definitions, diagnostic criteria, estimation of severity, and treatments," Diabetes Care, vol. 33, no. 10, pp. 2285-2293, 2010.

[6] B. C. Callaghan, H. T. Cheng, C. L. Stables, A. L. Smith, and E. L. Feldman, "Diabetic neuropathy: clinical manifestations and current treatments," The Lancet Neurology, vol. 11, no. 6, pp. 521-534, 2012.

[7] A. Sadosky, J. Mardekian, B. Parsons, M. Hopps, E. J. Bienen, and J. Markman, "Healthcare utilization and costs in diabetes relative to the clinical spectrum of painful diabetic peripheral neuropathy," Journal of Diabetes and its Complications, vol. 29, no. 2, pp. 212-217, 2015.

[8] T. Shiels, M. Abebaw, A. I. Bilal, and T. Tesfaye, "Treatment pattern and factors associated with blood pressure and fasting plasma glucose control among patients with type 2 diabetes mellitus in Police Referral Hospital in Ethiopia," Ethiopian Journal of Health Sciences, vol. 28, no. 4, pp. 461-472, 2018.

[9] G. Jember, Y. A. Melsew, B. Fisseha, K. Sany, A. Y. Gelaw, and B. Janakiraman, "Peripheral sensory neuropathy and associated factors among adult diabetes mellitus patients in Bahr Dar, Ethiopia," Journal of Diabetes \& Metabolic Disorders, vol. 16, no. 1, 2017.

[10] A. J. Boulton, "Guidelines for diagnosis and outpatient management of diabetic peripheral neuropathy. European Association for the Study of Diabetes, Neurodiab," Diabetes \& Metabolism, vol. 24, 1998.

[11] A. J. Boulton, R. A. Malik, J. C. Arezzo, and J. M. Sosenko, "Diabetic somatic neuropathies," Diabetes Care, vol. 27, no. 6, pp. 1458-1486, 2004.

[12] B. J. Shea, B. C. Reeves, G. Wells et al., “AMSTAR 2: a critical appraisal tool for systematic reviews that include randomised or non-randomised studies of healthcare interventions, or both," BMJ, vol. 358, article j4008, 2017.

[13] A. Liberati, D. G. Altman, J. Tetzlaff et al., "The PRISMA statement for reporting systematic reviews and meta-analyses of studies that evaluate health care interventions: explanation and elaboration," Annals of Internal Medicine, vol. 151, no. 4, pp. W-65-W-94, 2009. 
[14] J. P. Higgins, S. G. Thompson, J. J. Deeks, and D. G. Altman, "Measuring inconsistency in meta-analyses," BMJ, vol. 327, no. 7414, pp. 557-560, 2003.

[15] D. Worku, L. Hamza, and K. Woldemichael, "Patterns of diabetic complications at Jimma University Specialized Hospital, southwest Ethiopia," Ethiopian Journal of Health Sciences, vol. 20, no. 1, pp. 33-39, 2011.

[16] E. K. Gudina, S. T. Amade, F. A. Tesfamichael, and R. Ram, "Assessment of quality of care given to diabetic patients at Jimma University Specialized Hospital diabetes follow-up clinic, Jimma, Ethiopia," BMC Endocrine disorders, vol. 11, no. 1, 2011.

[17] A. Ejigu, "Brief communication: patterns of chronic complications of diabetic patients in Menelik II hospital, Ethiopia," Ethiopian Journal of Health Development, vol. 14, no. 1, pp. 113-116, 2000.

[18] T. Tsehay, E. Engidawork, and A. Ahmed, "Assessment of antidiabetic medication adherence and its determinants among ambulatory patients with type 2 diabetes at Tikur Anbessa Specialized Hospital, Addis Ababa, Ethopia," The Journal of Pharmacy and Alternative Medicine, vol. 11, 2016.

[19] A. A. Abejew, A. Z. Belay, and M. W. Kerie, "Diabetic complications among adult diabetic patients of a tertiary hospital in northeast Ethiopia," Advances in Public Health, vol. 2015, 7 pages, 2015.

[20] M. Gizaw, A. D. Harries, S. Ade et al., "Diabetes mellitus in Addis Ababa, Ethiopia: admissions, complications, and outcomes in a large referral hospital," Public Health Action, vol. 5, no. 1, pp. 74-78, 2015.

[21] Y. Feleke and F. Enquselassie, "An assessment of the health care system for diabetes in Addis Ababa, Ethiopia," Ethiopian Journal of Health Development, vol. 19, no. 3, pp. 203-210, 2005.

[22] S. Wild, G. Roglic, A. Green, R. Sicree, and H. King, "Global prevalence of diabetes: estimates for the year 2000 and projections for 2030," Diabetes Care, vol. 27, no. 5, pp. 1047-1053, 2004.

[23] B. B. Gebre and Z. M. Assefa, "magnitude and associated factors of diabetic complication among diabetic patients attending Gurage zone hospitals, South West Ethiopia," BMC Research Notes, vol. 12, no. 1, p. 780, 2019.

[24] A. T. Korsa, E. S. Genemo, H. G. Bayisa, and M. G. Dedefo, "Diabetes mellitus complications and associated factors among adult diabetic patients in selected hospitals of West Ethiopia," The Open Cardiovascular Medicine Journal, vol. 13, no. 1, pp. 41-48, 2019.

[25] R. Lebeta, Z. Argaw, and B. Walle, "Prevalence of diabetic complications and its associated factors among diabetes mellitus patients attending diabetes mellitus clinics; institution based cross sectional study," American Journal of Health Research, vol. 5, no. 2, p. 38, 2017.

[26] A. MB, Y. E, and T. G, "Diabetic complications among followup patients: a cross-sectional study at Jimma University Specialized Hospital diabetic clinic," Journal of Clinical and Molecular Endocrinology, vol. 3, no. 1, p. 45, 2018.

[27] T. Fiseha and A. G. Belete, "Diabetes mellitus and its associated factors among human immunodeficiency virus-infected patients on antiretroviral therapy in Northeast Ethiopia," BMC Research Notes, vol. 12, no. 1, article 372, 2019.

[28] T. AN, W. C, T. GM et al., "Major micro vascular complications and associated risk factors among diabetic outpatients in Southwest Ethiopia," Endocrinology \& Metabolic Syndrome, vol. 6, no. 4, p. 272, 2017.

[29] D. J. Tesfaye, "Coexistence of chronic complications among diabetic patients at Nigist Eleni Mohammed Memorial Hospital, Hosanna, south Ethiopia," Open Access Library Journal, vol. 2, no. 1, p. 1, 2015.

[30] G. Jarso, A. Ahmed, and Y. Feleke, "The prevalence, clinical features and management of periphral neuropathy among diabetic patients in Tikur Anbessa and St. Paul's Specialized University Hospitals, Addis Ababa, Ethiopia," Ethiopian medical journal., vol. 49, no. 4, pp. 299-311, 2011.

[31] F. T. Lester, "The clinical pattern of diabetes mellitus in Ethiopians," Diabetes Care, vol. 7, no. 1, pp. 6-11, 1984.

[32] G. Gill, A. Gebrekidan, P. English, D. Wile, and S. Tesfaye, "Diabetic complications, and glycaemic control in remote North Africa," QJM, vol. 101, no. 10, pp. 793-798, 2008.

[33] A. Fasil, B. Biadgo, and M. Abebe, "Glycemic control and diabetes complications among diabetes mellitus patients attending at University of Gondar Hospital, Northwest Ethiopia," Diabetes, Metabolic Syndrome, and Obesity: Targets and Ther$a p y$, vol. 12, pp. 75-83, 2019.

[34] F. T. Lester, "Long-standing diabetes mellitus in Ethiopia: a survey of 105 patients," Diabetologia, vol. 25, no. 3, pp. 222-225, 1983.

[35] F. T. Lester, J. Abdulkadir, D. Larson, and P. Quana'a, "Diabetes mellitus: clinical features in 404 Ethiopians," Ethiopian Medical Journal, vol. 14, no. 4, pp. 185-198, 1976.

[36] W. Teshager, Prevalence of depression, and associated factors among adult diabetic patients attending outpatient department, at Felege Hiwot Referral Hospital, Bahir Dar, Northwest Ethiopia, 2016, Addis Ababa University, 2016.

[37] G. Kefeany, Status of vitamin B12 level among type 2 diabetes mellitus patients on metformin treatment attending diabetic clinic of the Tikur Anbessa Specialized Hospital, Addis Ababa, Ethiopia, Addis Ababa University, 2017.

[38] T. Kassahun, T. Eshetie, and H. Gesesew, "Factors associated with glycemic control among adult patients with type 2 diabetes mellitus: a cross-sectional survey in Ethiopia," $B M C$ Research Notes, vol. 9, no. 1, article 78, 2016.

[39] T. Eticha, A. Mulu, H. Gebretsadik, G. Kahsay, and D. Y. Ali, "Factors associated with poor glycemic control in type 2 diabetic patients investigated at Ayder Referral Hospital, Mekelle, Ethiopia," International Journal of Pharmacy and Pharmaceutical Research (IJPPR), vol. 6, no. 3, pp. 160-171, 2016.

[40] W. Cheneke, S. Suleman, T. Yemane, and G. Abebe, “Assessment of glycemic control using glycated hemoglobin among diabetic patients in Jimma University Specialized Hospital, Ethiopia," BMC Research Notes, vol. 9, no. 1, p. 96, 2016.

[41] T. G. Mariam, A. Alemayehu, E. Tesfaye et al., "Prevalence of diabetic foot ulcer and associated factors among adult diabetic patients who attend the diabetic follow-up clinic at the University of Gondar Referral Hospital, North West Ethiopia, 2016: institutional-based cross-sectional study," Journal of diabetes research., vol. 2017, pp. 1-8, 2017.

[42] G. T. Demoz, A. Gebremariam, H. Yifter et al., "Predictors of poor glycemic control among patients with type 2 diabetes on follow-up care at a tertiary healthcare setting in Ethiopia," BMC Research Notes, vol. 12, no. 1, p. 207, 2019.

[43] D. M. Yigazu and T. A. Desse, "Glycemic control and associated factors among type 2 diabetic patients at Shanan Gibe Hospital, Southwest Ethiopia," BMC Research Notes, vol. 10, no. 1, p. 597, 2017. 
[44] M. Alebachew Woldu and C. Diriba Wami, "Factors associated with poor glycemic control among patients with type 2 diabetes mellitus in Ambo Hospital, Ambo; Ethiopia," Endocrinology \& Metabolic Syndrome, vol. 3, no. 4, 2014.

[45] D. Abdissa, T. Adugna, U. Gerema, and D. Dereje, "Prevalence of diabetic foot ulcer and associated factors among adult diabetic patients on follow-up clinic at Jimma Medical Center, Southwest Ethiopia, 2019: an institutional-based crosssectional study," Journal of Diabetes Research, vol. 2020, Article ID 4106383, 6 pages, 2020.

[46] K. Tamru, F. Aga, E. Berhanie, Y. A. Aynalem, and W. S. Shiferaw, "Incidence of diabetic nephropathy in patients with type 2 diabetes mellitus at a tertiary healthcare setting in Ethiopia," Diabetes \& Metabolic Syndrome: Clinical Research \& Reviews., vol. 14, no. 5, pp. 1077-1083, 2020.

[47] D. Abdissa, N. Hamba, K. Kene et al., "Prevalence and determinants of peripheral neuropathy among type 2 adult diabetes patients attending Jimma University Medical Center, Southwest Ethiopia, 2019, an Institutional-Based Cross-Sectional Study," Journal of Diabetes Research, vol. 2020, Article ID 9562920, 8 pages, 2020.

[48] G. T. Woldemariam, N. T. Atnafu, Y. T. Radie et al., "Determinants of diabetic foot ulcer among adult patients with diabetes attending the diabetic clinic in Tikur Anbessa Specialized Hospital, Addis Ababa, Ethiopia: unmatched case-control study," Diabetes, Metabolic Syndrome, and Obesity: Targets and Therapy, vol. 13, pp. 3739-3747, 2020.

[49] M. YimamAhmed, S. H. Ejigu, A. Z. Zeleke, and M. Y. Hassen, "Glycemic control, diabetes complications, and their determinants among ambulatory diabetes mellitus patients in Southwest Ethiopia: a prospective cross-sectional study," Diabetes, Metabolic Syndrome, and Obesity: Targets and Therapy, vol. 13, pp. 1089-1095, 2020.

[50] S. Sobhani, H. Asayesh, F. Sharifi et al., "Prevalence of diabetic peripheral neuropathy in Iran: a systematic review and metaanalysis," Journal of Diabetes \& Metabolic Disorders, vol. 13, no. 1, p. 97, 2014.

[51] A. Vasigh, A. Abdi, M. Borji, and A. Tarjoman, "The prevalence of neuropathy among type 1 diabetic adolescents in Iran: a systematic review and meta-analysis," International Journal of Adolescent Medicine and Health, 2019.

[52] U. M. Abdel-Motal, E. M. Abdelalim, H. Abou-Saleh, and H. Zayed, "Neuropathy of type 1 diabetes in the Arab world: a systematic review and meta-analysis," Diabetes Research and Clinical Practice, vol. 127, pp. 172-180, 2017.

[53] R. de Souza, D. Debiasi, L. B. Ceretta, P. W. Simões, and L. Tuon, "Meta-analysis and meta-regression of the prevalence of diabetic peripheral neuropathy among patients with type 2 diabetes mellitus," International Archives of Medicine, vol. 9, 2016.

[54] W. S. Shiferaw, T. Y. Akalu, Y. Work, and Y. A. Aynalem, "Prevalence of diabetic peripheral neuropathy in Africa: a systematic review and meta-analysis," BMC Endocrine Disorders, vol. 20, no. 1, p. 49, 2020.

[55] J. Sun, Y. Wang, X. Zhang, S. Zhu, and H. He, "Prevalence of peripheral neuropathy in patients with diabetes: a systematic review and meta-analysis," Primary Care Diabetes, vol. 14, no. 5, pp. 435-444, 2020.

[56] G. Said, "Diabetic neuropathy-a review," Nature Clinical Practice Neurology, vol. 3, no. 6, pp. 331-340, 2007. 